\title{
Percepción de necesidades básicas, ¿es necesaria una consulta de enfermería para pacientes en hemodiálisis?
}

\author{
David Alba de la Torre, Marta Moreda Díaz-Pavón, Raquel Fernández Camacho, Miriam Fernández \\ Rodríguez, Maite Barahona Muñoz
}

Hospital Universitario de Getafe y Hospital Universitario Ramón y Cajal. Madrid

\section{Introducción:}

La necesidad de atención holística a los pacientes ha sido un tema recurrente desde la antigua Grecia, hasta su auge en el siglo XX. En una época marcada por la especialización médica, atender al paciente de forma integral sigue siendo vital. Actualmente se ha desarrollado el concepto de Calidad de Vida Relacionada con la Salud (CVRS), que ha demostrado ser la mejor herramienta para la atención integral del paciente con enfermedad crónica. En la Enfermedad Renal Crónica, la CVRS ha demostrado un menoscabo en todas las dimensiones de la calidad de vida de los pacientes. Esto habla de la necesidad de atenderlos de forma integral y muestra de ello son la cantidad de publicaciones que relacionan la enfermedad renal con las relaciones familiares, el empleo, el sexo o la alimentación.

\section{Objetivo, material y métodos:}

El objetivo del estudio era responder a una simple cuestión, ¿Conocemos realmente las necesidades de nuestros pacientes? Para contestar, escogimos una muestra de 124 pacientes en estadío V de ERC en tratamiento con hemodiálisis y 50 enfermeras/os de las mismas unidades. Se administró a los pacientes un cuestionario propio adaptado a partir del SF-36, que incluía 7 de las 8 dimensiones de salud. Sirve para relacionar la interferencia de la enfermedad y su tratamiento con la adaptación funcional y la calidad de vida del paciente. Es un cuestionario autoadministrado, con un índice de fiabilidad alto, que recoge 4 datos sociodemográficos y evalúa 16 ítems tipo "Likert" con 5 posibles respuestas. A enfermería se le entregó el mismo cuestionario, para evaluar las diferencias entre los datos proporcionados por los pacientes y lo que perciben los profesionales. Una vez obtenidas las encuestas se pasaron los resultados a una escala numérica de 0 a 100 para proceder a su comparación. Además, se entregó un segundo cuestionario a los profesionales en el que tenían que relacionar cada uno de esos 16 ítems con el grupo sociodemográfico que ellos creían más afectado en cada caso (Por ejemplo para la alimentación, varón, mayor de 85 años, jubilado y viudo). Éste, nos permitía saber si los profesionales son capaces de reconocer los problemas que más afectan a cada paciente atendiendo a sus datos sociales.

\section{Conclusiones:}

Los primeros datos recogidos entre las encuestas de los pacientes, mostraban una alta dispersión lo que nos da una idea de que la atención ha de ser individualizada. Cada paciente presenta unos problemas particulares que responden a su situación personal y que pueden salirse de la tendencia sociodemográfica de cada ítem. Creemos que es difícil evaluar los problemas biopsicosociales que tiene un paciente atendiendo solo a datos sociodemográficos, a pesar del conocimiento del profesional. La alta dispersión, hace que no se genere una tendencia clara y tratar de evaluar a los pacientes durante su sesión no es posiblemente lo más adecuado, debido a la presencia de otros pacientes y a la intimidad que requieren algunos temas. Facilitar un entorno adecuado que permita al paciente exponer todos sus problemas sin reparos, es fundamental para realizar una atención holística real. 


\section{Referencias Bibliográficas}

1. Predictores psicosociales de la calidad de vida en pacientes con insuficiencia renal crónica en tratamiento de hemodiálisis. Carmen M. Perales-Montilla, Ana García-León, Gustavo A. Reyes del Paso. Revista Nefrología. 2012;32(5): pag.622-630.

2. Factores psicosociales relacionados con la calidad de vida en salud en pacientes hemodializados. Alfonso Urzúa, Ruth Pavlov, Vanessa Pino. Terapia Psicológica. 2011, vol. 29, N¹1, pag. 135-140.

3. Diferencias en la calidad de vida relacionada con la salud entre hombres y mujeres en tratamiento en hemodiálisis. I. Vazquez, F. Valderrábano, J. Fort, R. Jofré, J. M. López-Gómez, F. Moreno, D. Sanz-Guajardo. Nefrología. 2004, Vol. 24, N², pag. 167-177.

4. Calidad de vida en pacientes con insuficiencia renal crónica en tratamiento con diálisis. Anna Seguí Gomà, Patricia Amador Peris, Ana Belén Ramos Alcario. Revista de la sociedad española de enfermería nefrológica. 2010; 13(3): pag. 155-160.

5. Asociación entre dependencia funcional y sintomatología afectivo-depresiva en pacientes en programa de hemodiálisis. María Mena Mayayo, Eva Cerdán Urrutia, Ana Pérez-Seoane Justo, Joaquín Manrique Escola. Revista de la sociedad española de enfermería nefrológica. 2010; 13(4): pag. 236-241.

6. Depression in patients on hemodialysis and their caregivers. Zeb Saeed, Aizaz M. Ahmad, Abdul Shakoor, Farkhanda Ghafoor, Shumaela Kanwal. Saudi Journal of Kidney Diseases anda Transplantation. 2012; 23(5): pag. 946-952.

7. Pre-dialysis patients' perceived autonomy, selfesteem and labor participation: associations with illness perceptions and treatment perceptions. A cross-sectional study. Daphe L. Jansen, Diana C. Grootendorst, Mieke Rijken, Monique Heijmans, Ad A. Kaptein, Elisabeth W. Boeschoten, Friedo W. Dekker. BMC nephrology. 2010, 11:35.

8. La metodología de cuidados en el abordaje al cansancio del cuidador del paciente incidente en programa de hemodiálisis. Trinidad López Sánchez, $\mathrm{M}^{\mathrm{a}}$ Luz Amoedo Cabrera, Francisco Javier Toro Prieto, Marta Pérez Doctor, M ${ }^{a}$ Carmen Chozas López. Revista de la sociedad española de enfermería nefrológica. 2010; 13(3): pag. 190-194.

9. Características del cuidado familiar a pacientes dependientes en programa de hemodiálisis. Lola Andreu Periz, Carmen Moreno Arroyo, Maricel Julve Ibañez. Revista de la sociedad española de enfermería nefrológica. 2011; 14(1): pag. 51-52.

10. Dialysis and sexuality. Donna Beal-Lloyd, Carla J. Groh. American Nephrology Nurses' Associations. 2012; 39(4): pag: 281-283.

11. Modelos y teorías en enfermería. Ann Marriner Tomey, Martha Raile Alligood. Ed. Elsevier. Madrid. 2007.

12. Atención avanzada al paciente renal, cuidados nefrológicos Vol (4). Pilar Peña Amaro, Juan Gómez Salgado, Antonio Barranco Martos, Francisco García Alcaraz. Ed. Enfo. Madrid. 2008.

13. 500 cuestiones que plantea el cuidado del enfermo renal. Lola Andreu Periz, Enriqueta Force Sanmartín. Ed. Masson. Barcelona. 2001.

14. Korshunova G. Kirilenko. ¿Qué es la filosofía? En los hontanares del saber filosófico. Moscú: Editorial Progreso; 1989. 